\title{
Aberration-Corrected Four-Detector STEM-EDS Analysis of Embedded Nanoclusters
}

\author{
Chad M. Parish and Michael K. Miller
}

Physical Sciences Directorate, Oak Ridge National Laboratory, Oak Ridge, TN 37831, USA

Examining small particles embedded in a specimen foil is a challenging application for analytical STEM methods. When the particles are $\sim 2 \mathrm{~nm}$ or smaller and the foils must be prepared by FIB (which results in thicker foils and more surface damage), the challenges increase. Here we report the use of aberrationcorrected STEM, equipped with high-detection-efficiency EDS systems, and multivariate statistical analysis (MVSA) post-treatment analysis of the data, to analyze such particles.

Nanostructured ferritic alloys (NFAs) require advanced analytical STEM methods. NFAs are Fe-Crbased steels that contain high densities $\left(>10^{23} / \mathrm{m}^{3}\right)$ of small $(<2 \mathrm{~nm})$ oxygen- and vacancy-enriched precipitates, referred to as nanoclusters (NCs) [1]. In alloy 14YWT, Ti-Y-based NCs impart creep strengths several orders of magnitude superior to conventional steels, and NCs are hypothesized to provide tremendous tolerance to neutron irradiation in reactors. Because $\mathrm{Fe}-\mathrm{Cr}$ alloys are ferromagnetic, FIB-preparation is helpful to reduce aberrations of the electron beam in a STEM column. Neutronirradiated specimens should be FIBed to reduce the specimens' radioactivity hazard and gamma-induced EDS dark counts. Due to non-uniform dose profiles, ion-irradiated specimens should be FIBed to find the desired dose depth in a large irradiated coupon. Because even the best FIB specimens of stainless steels are typically 20 60 $\mathrm{nm}$ thick and passivated with Fe-Cr-oxide on the top and bottom faces, STEM experiments are less ideal than in, for instance, ion-milled cross-sections of epitaxial films. Identifying a single 2-nm O- and vacancy-enriched $\mathrm{NC}$ in a $20 \sim 60 \mathrm{~nm}$ foil requires high signal levels. High spatial resolution requires a small probe size. The $200+\mathrm{keV}$ electron probes cause sputtering damage of the foil [2,3]. Maximizing the acquired signal at a given probe size requires high-efficiency detectors, bright sources, and aberration-corrected optics, which are available on FEI Titan platforms with ChemiSTEM technology [4]. Even with this advanced tool, signal levels will still be low in absolute terms, and MVSA methods [5] are needed to clearly identify features, such as NCs and grain boundary segregation.

We examined ion-irradiated 14YWT NFA [1] in the North Carolina State University FEI Titan with ChemiSTEM technology, using the four-detector EDS system to acquire spectrum images, which were then interrogated with the Sandia Laboratory AXSIA [5] code. In an unirradiated region, NCs $\sim 1 \mathrm{~nm}$ or smaller are visible in both on-zone and off-zone oriented grains (Figure 1). Grain-boundary (GB) solute enrichment is observed (and likely influences the creep properties), and a distribution of small $(<1 \mathrm{~nm})$ to large $(>5 \mathrm{~nm}) \mathrm{NCs}$ are observed. In a cryo-irradiated region of the same specimen $(5 \sim 10 \mathrm{dpa}$ [displacements per atom] radiation damage), few NCs survive the cryo-irradiation, having been ballistically mixed into the matrix (Figure 2). The W-enriched GB component (MVSA\#2) in Figure 2 is broader than in Figure 1, and indicates small levels of W enrichment. Combined high-efficiency EDS and MVSA identified the GB component even after dispersal by the irradiation. These microstructural insights are only possible due to the combination of high-efficiency aberration-corrected EDS and MVSA data reduction [6].

[1] M. K. Miller and C. M. Parish,Mater. Sci Tech., 27[4] (2011) p. 729.

[2] R. F. Egerton et al., Ultramicrosc., 110 (2010) p. 991. 
[3] C. M. Parish and M. K. Miller, Microsc. Microan., in press.

[4] P. G. Kotula et al., Microsc. Microan., 18[4] (2012) p. 691.

[5] P. G. Kotula et al., Microsc. Microan., 9[1] (2003) p. 1.

[6] Research sponsored by the Materials Sciences and Engineering Division, Office of Basic Energy Sciences, U.S. Department of Energy. We acknowledge the use of the Analytical Instrumentation Facility (AIF) at North Carolina State University, which is supported by the State of North Carolina and the National Science Foundation.
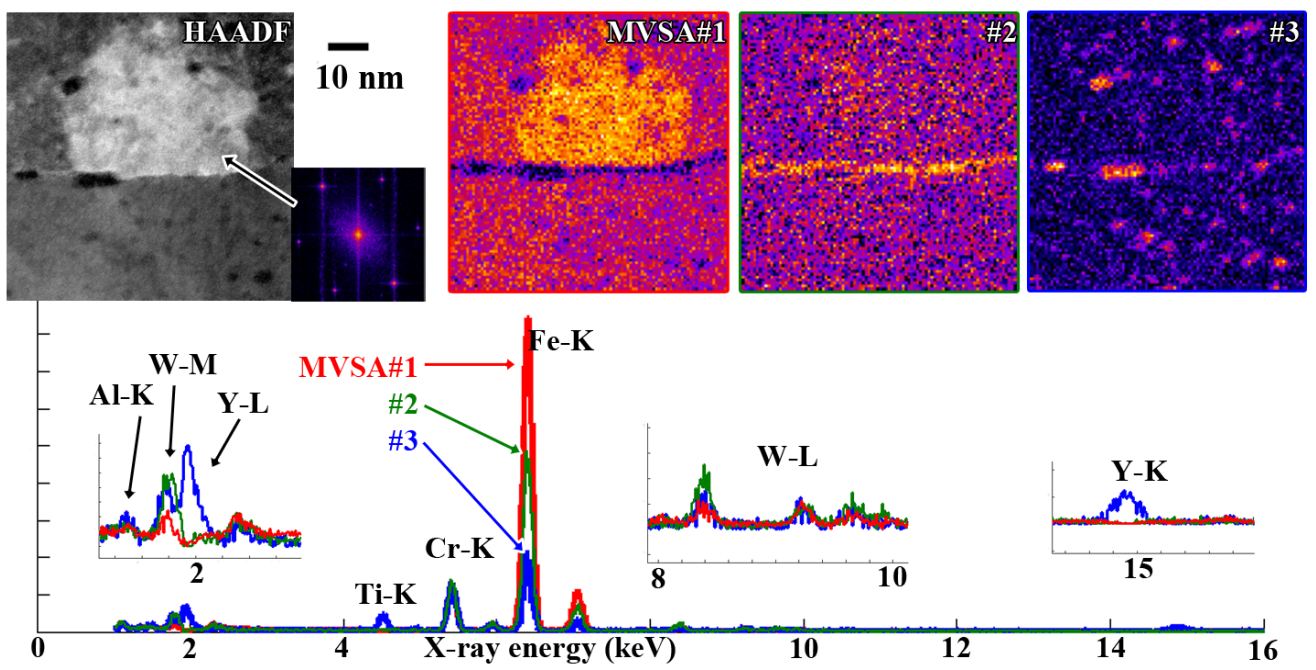

Figure 1: Left, STEM-HAADF image of several grains in NFA. Inset indicates the bright grain is at $<111>$ orientation. Right, MVSA of EDS spectrum image, showing three components. Third component (blue outline and blue EDS loading spectrum) is Ti-Y-rich NCs with sizes $\sim 1 \mathrm{~nm}$ detected in the $20 \sim 50 \mathrm{~nm}$-thick foil.
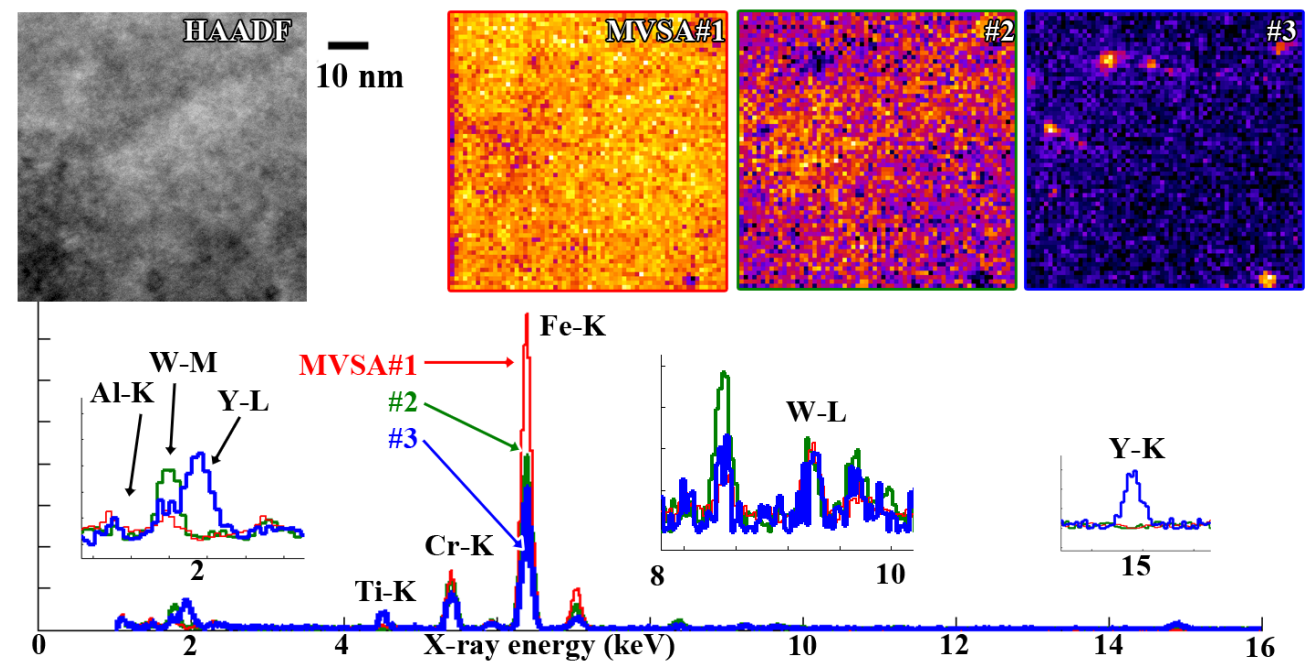

Figure 2: Left, STEM-HAADF image of several grains in cryo-irradiated NFA. Right, MVSA of EDS spectrum image, showing three components. Second component (green outline and EDS loading spectrum) is a diffuse grain boundary indicting blurring of the sharp segregant profile. Third component (blue outline and EDS loading spectrum) shows a low density of NCs, indicating ballistic dissolution of many NCs leaving only remnants of the larger particles. 